\title{
ROBOTS, INTELIGENCIA ARTIFICIAL Y REALIDAD VIRTUAL: UNA APROXIMACIÓN EN EL SECTOR DEL TURISMO ${ }^{1}$
}

\author{
María del Mar Alonso Almeida \\ Universidad Autónoma de Madrid \\ https://orcid.org/0000-0003-4957-3689
}

\section{RESUMEN}

A pesar de los cambios producidos por las tecnologías de la información e internet en el sector turismo, los pronósticos apuntan a que una nueva revolución se va a producir propiciada por la introducción de robots, inteligencia artificial y realidad virtual en las industrias turísticas. En la actualidad, la adopción de estas tecnologías está en un estadio muy inicial pero la investigación realizada en otros sectores apunta a que las mismas podrían también tener un gran impacto en las operaciones turística.

Palabras clave: Robot, inteligencia artificial; realidad virtual; turismo.

\section{Robots, artificial intelligence and virtual reality: An approach to tourism industries}

\section{ABSTRACT}

In spite of the changes produced by the information technologies and internet in the tourism sector, the forecasts point out a new revolution produced by the introduction of robots, artificial intelligence and virtual reality in the tourist industries. Currenly, the adoption of these technologies is in their infancy but the research carried out in other sectors suggests that they also could have a great impact on the tourism.

Keywords: Robots, virtual Intelligence; virtual reality; tourism.

Fecha de recepción: 18 de marzo de 2018

Fecha de aceptación: 12 de diciembre de 2018

Facultad de Ciencias Económicas y Empresariales. Universidad Autónoma de Madrid. 28049 MADRID (España).E-mail: mar.alonso@uam.es

1 Este trabajo está financiado por la $5^{\text {a }}$ Convocatoria de "Proyectos de Cooperación Interuniversitaria UAM-BANCO SANTANDER CON ASIA”, código (ASIA01). La Investigadora Principal es María del Mar Alonso Almeida. 


\section{INTRODUCTION}

El sector del turismo ha sufrido transformaciones radicales en las últimas décadas debido a las tecnologías de información y comunicaciones e internet (TICs) (Manero et al., 2011). La irrupción de las TICs ha supuesto cambios no sólo a nivel meso respecto a la estructura del propio sector y la forma de relacionarse o la aparición de nuevos competidores y sustitutos, sino que a nivel micro, las empresas de todos los subsectores turísticos se han visto afectadas de alguna manera, lo que les ha obligado a adaptar su cadena de valor para amoldarse a esta nueva realidad y no perder competitividad (Rodriguez Antón et al., 2008).

Las últimas tendencias en la industria del turismo hablan de una nueva era en el uso de la tecnología en el turismo que se basarán en la utilización de tecnologías de la información complejas como el uso de robots; el big data y la inteligencia artificial y la realidad virtual (Bowen y Whalen, 2017).

La incorporación de robots ha sido una práctica habitual en la industria manufacturera en las últimas décadas, sin embargo, en los sectores de servicios el uso de robots e inteligencia artificial está en un estado embrionario. Ahora bien, la poca literatura científica sobre la adopción de este tipo de tecnología en estas industrias, sugiere que la introducción de robots puede suponer una revolución en la industria de los viajes y el turismo tanto en lo que tiene que ver con los puestos de trabajo, como las operaciones y la calidad del servicio, lo que va provocar transformación en los destinos y en la industria en su conjunto.

Además, en la actualidad, los turistas dejan trazas o huellas electrónicas en internet, incluso desde antes de viajar, cuando buscan información sobre los productos y destinos, durante el desarrollo del viaje y al finalizar el mismo tanto mediante la navegación en internet o en un dispositivo móvil, como mediante sus consumos y pagos y por proveer informaciones, opiniones, evaluaciones, fotos y otro material multimedia en sus redes sociales (Alonso-Almeida et al., 2016; 2018). En consecuencia, existe una enorme cantidad de información sobre los viajeros, su comportamiento de viaje, sus necesidades y la experiencia obtenida tanto con el destino como con los productos turísticos almacenada en las diversas fuentes de datos, de naturaleza heterogénea, que en la actualidad está todavía poco explotadas.

Sin embargo, para encontrar relaciones causales entre las diferentes fuentes de datos -big data- es necesario aplicar técnicas de inteligencia de negocio y minería de datos. Así, la inteligencia artificial mediante la combinación de todos los rastros e información provista de forma deliberada puede obtener una información personalizada para cada turista $\mathrm{y}$, como consecuencia, proveerle de la información de viaje que necesita en cada momento (Rodriguez-Antón et al., 2008; Bowen y Whalen, 2017).

Finalmente, la realidad virtual introduce nuevos elementos emocionales y sociales en el sector del turismo; cambiando la forma de relacionarse entre compradores y vendedores, transformando la forma de viajar y cambiando las necesidades de los consumidores, que ya no sólo buscan comprar sino "vivir experiencias" (Alonso-Almeida, 2018).

Esta revolución presenta oportunidades importantes en términos económicos, eficiencia y competitividad, pero también amenazas. Como consecuencia, es necesario adelan- 
tarse a este proceso de robotización en las industrias de viajes y turismo y analizar las implicaciones económicas, operativas y sociales de la incorporación de los robots a los viajes y turismo en su conjunto.

Los objetivos del presente trabajo consisten en poner de relieve cómo se encuentra el estado del arte y la penetración de los temas propuestos en el sector del turismo y poner este conocimiento a disposición del sector del turismo para que las empresas y otros organismos puedan diseñar los diferentes escenarios que se podrán desarrollar y sus impactos en el turismo y los investigadores avanzar en este ámbito.

\section{LITERATURA REVIEW}

\subsection{Robots en turismo}

Una de las tendencias más innovadoras y disruptivas es el uso de robots en las industrias de servicios (Bowen y Whalen, 2017). En un futuro cercano, sobrepasando cualquier película de ciencia ficción, las personas van a convivir con robots, androides y otras formas de inteligencia artificial cada vez más sofisticadas (Santos, 2017). De acuerdo con esta autora, la tecnología ha pasado por diversas fases, primero las personas se conectaban a internet, ahora son las cosas las que conectan a internet para mejorar la vida de las personas. La siguiente fase, que es inminente, va consistir en que los robots interactúen con el entorno de manera autónoma e independiente del control humano, con la posibilidad de que incluso las personas se combinen con robots para mejorarse.

Los pronósticos en el corto plazo es que el mercado global de la robótica industrial crecerá aun 56,9\& en el periodo entre 2017 y 2023 (Resarch and Markets, 2017). Aunque en los sectores de servicios esta introducción es todavía muy nueva y tímida. Existen iniciativas pioneras en sectores como la educación (Timms, 2016), almacenaje y logística (Maurer et al., 2016), banca e inversión (Dunis et al., 2017), medicina y teleasistencia (Melendez-Fernandez et al., 2017) y el turismo y los viajes (Bowen y Whalen, 2017; Ivanov y Webster, 2017).

Santos (2017) explica que la Unión Europea es consciente de la importancia que en el futuro cercano pueden tener los robots a nivel europeo y ha elaborado un informe con fecha del 31 de mayo de 2016 en que se recogen recomendaciones sobre normas de Derecho con el fin de "asegurar que los robots estén y sigan estando al servicio de los seres humanos". Este informe ha sido aprobado el 16 de febrero de 2017 por el Parlamento Europeo y recoge las principales líneas de trabajo que en términos legales deberían desarrollarse en el futuro. Entre otras iniciativas está la creación de una Agencia Europea de Robótica e inteligencia artificial y la elaboración de un Código de Conducta ética voluntario que sirva de base para regular quién será responsable de los impactos sociales, ambientales y de salud humana de la robótica.

De hecho, uno de los ámbitos que se pueden ver más afectados por los robots es el mercado de trabajo. En la Unión Europea la población está envejecida y los nacimientos no son capaces de cubrir las necesidades de mano de obra generacionales. Además, un estudio realizado por la Fundación Cotec (2017) mostró que un 66,6\% de los encuestados pensaban que muchos o bastantes puestos de trabajo serán sustituidos por robots $\mathrm{u}$ 
ordenadores, especialmente en tareas rutinarias. Por tanto, la robotización puede tener un alto impacto en la industria de los viajes y el turismo, industrias que son intensivas en mano de obra, con muchos perfiles con baja cualificación, trabajos repetitivos y largas jornadas de trabajo. Los robots inteligentes podrán ser multidisciplinares y podrán sustituir a personas realizando tareas domésticas, vigilancia, seguridad, transporte o cuidado personal.

Por tanto, en el caso del sector de viajes y turismo podría ocurrir como ha ocurrido en la industria manufacturera, que los empleos de nivel inicial, particularmente tareas repetitivas y no especializados, serán gradualmente reemplazados por robots, mientras que las personas complementarán estos puestos de trabajo realizando los trabajos de mayor valor añadido, lo que llevará a una mejora del puesto de trabajo, incremento salarial y mayor flexibilidad, además de nuevos puestos de trabajo de mayor cualificación porque estos cambios conllevan, nuevos conocimientos y capacidades (Acemoglu y Restrepo, 2018). Además, en concreto en el sector de viajes y turismo puede ver solucionado el problema del empleo estacional.

Algunos autores señalan que ya existen algunos robots en el sector en los países asiáticos, de forma marginal, robots camareros, robots cocineros o robots acompañantes. Por poner algún ejemplo pionero, el hotel Henn-na Hotel en Japón, es conocido porque su recepción es atendida por robots (Bowen and Whalen, 2017). Los hoteles Aloft tienen robots en el servicio de habitaciones para las peticiones de los huéspedes (Hertzfield, 2016). Ivanov et al. (2017) también cita otras formas en que se pueden usan los robots en el sector de viajes: como porteadores; para el check-in o check-out; como asistentes de cabina; como ayudantes en una reunión, o un evento o un restaurante; recogida y entrega del equipaje en aeropuertos; y como guías en museos entre otros, aunque todavía no se están utilizando.

Así, sin ánimo de ser exhaustivo los robots podrían proporcionar multitud de beneficios al sector de viajes y turismo (ver tabla 1).

\section{Tabla 1 \\ BENEFICIOS DE LOS ROBOTS EN EL SECTOR DE VIAJES Y TURISMO}

\begin{tabular}{ll}
\hline $\begin{array}{l}\text { Ahorro de costes e incre- } \\
\text { mento de productividad }\end{array}$ & $\begin{array}{l}\text { Operan 24 horas 7 días de la semana. } \\
\text { Pueden atender a distintos clientes a la vez. } \\
\text { No tienen limitación de idiomas, pueden atender en el idioma que } \\
\text { tengan incorporado. }\end{array}$ \\
\hline Mejora de la calidad de & $\begin{array}{l}\text { Eliminación o minimización de los errores. } \\
\text { servicio }\end{array}$ \\
& $\begin{array}{l}\text { Eliminación para las personas de tareas repetitivas. } \\
\text { Eliminación para los humanos de tareas peligrosas. } \\
\text { Minimización de quejas y de problemas de salud laboral en el } \\
\text { trabajo. }\end{array}$ \\
\hline Mejora de la calidad del & $\begin{array}{l}\text { Las personas humanas se centran en las tareas y actividades de } \\
\text { valor. }\end{array}$ \\
& $\begin{array}{l}\text { Puestos de trabajo de mayor cualificación. } \\
\text { Nuevos puestos de trabajo. }\end{array}$ \\
\hline
\end{tabular}


Innovación

\author{
Servicios personalizados. \\ Servicios de alto valor añadido. \\ Mejora de los ingresos.
}

Fuente: Elaboración propia basado en información proporcionada en Hertzfield (2016); Bowen and Whalen (2017) y Ivanov et al. (2017).

Sin embargo, como apunta Kuo et al., (2017) la introducción de robots en el sector requiere que se 1) definan que servicios se pueden prestar; 2) cómo se va a interactuar con los clientes; 3) que nuevos socios se necesitan en la cadena de valor; 4) cómo va a ser el nuevo modelo de negocio; 5) cómo va a ser el nuevo diseño organizativo y 6) como a ser el desarrollo y entrega del servicio. Además, puede existir una serie de barreras o resistencias a la puesta en marcha de robots en el sector tanto por parte de los empleados como de los clientes, falta de visión estratégica por parte de la dirección, choque con la cultura organizativa o las propias características de la empresa puede suponer una barrera para el cambio (Ivanov y Webster, 2017).

Así, de momento hay más incógnitas que respuestas en esta tendencia y es evidente que se necesita más investigación multi e interdisciplinar en este ámbito para tratar de pronosticar su alcance e impacto en el sector de viajes y turismo.

\title{
2.2. Big data e inteligencia artificial
}

Como se ha apuntado en la introducción, en las últimas décadas con la eclosión de internet y redes sociales, grandes volúmenes de información existen de cada usuario, los cuales adecuadamente tratados y explotados pueden proporcionar información muy valiosa para los destinos y empresas turísticas, para identificar las necesidades y deseos conscientes y latentes de los turistas. Otra fuente que en los últimos años se ha sumada a las anteriores son los datos de geolocalización entre otros que se obtienen a través de los teléfonos móviles inteligentes.

Estas nuevas fuentes de información hacen necesario nuevos métodos de análisis para sacar el mayor partido posible a este big data. Capriello et al. (2013) utiliza tres métodos distintos analizar cuantitativamente grandes volúmenes de datos cualitativos de una forma relativamente eficiente y confiable; 1) mediante la codificación manual a través de los likes y dislikes; 2) mediante el análisis semántico de los mensajes dejados por los turistas y 3) mediante el denominado análisis stance-shift que es un tipo de maximiza la comprensión de la comunicación en línea a través de la identificación de patrones de lenguaje clave que destacan la evaluación del consumidor, las actitudes y determinar las futuras intenciones. Estos autores aplicaron los tres tipos de análisis al turismo rural y encontraron que los tres métodos eran adecuados para analizar este tipo de turismo.

Otro método que está ganando popularidad por su alta efectividad más allá del análisis de la semántica del texto es el análisis de sentimientos (sentimental analysis). Ese análisis permite identificar lo que el usuario siente de un sitio en particular a través de la calificación de las expresiones que usa en positivas, negativas o neutras (Chen et al., 2017). En los 
últimos años esta línea de investigación está avanzando aplicándose a distintos contextos. La tabla 2 muestra la investigación principal en este ámbito.

Tabla 2

INVESTIGACIÓN REALIZADA EN EL SECTOR DEL TURISMO UTILIZANDO SENTIMENTAL ANALYSIS

\begin{tabular}{|c|c|c|c|}
\hline Autores & $\begin{array}{c}\text { Año de } \\
\text { publicación }\end{array}$ & $\begin{array}{l}\text { Sector de } \\
\text { aplicación }\end{array}$ & Datos analizados \\
\hline Zhang et al. & 2011 & Restaurantes & $\begin{array}{l}\text { Comentarios en cantonés } \\
\text { sobre restaurantes }\end{array}$ \\
\hline Duan et al. & 2015 & Hoteles & $\begin{array}{l}\text { Comentarios de clientes } \\
\text { de } 81 \text { hoteles de Washing- } \\
\text { ton en el periodo de } 1999 \\
\text { a } 2011\end{array}$ \\
\hline Gonzalez-Rodriguez et al. & 2016 & Destino & $\begin{array}{l}200 \text { comentarios en ciao } \\
\text { sobre Barcelona }\end{array}$ \\
\hline Philander y Zhong & 2016 & Hoteles & Comentarios de twitter \\
\hline Gan et $a l$. & 2017 & Restaurantes & $\begin{array}{l}\text { Comentarios sobre restau- } \\
\text { rantes y bares de la base } \\
\text { Yelp.com }\end{array}$ \\
\hline Chen et al. & 2017 & Destino & $\begin{array}{l}\text { Comentarios de turistas } \\
\text { chinos sobre España }\end{array}$ \\
\hline Gitto y Mancuso & 2017 & Aeropuertos & $\begin{array}{l}\text { Comentarios de usuarios } \\
\text { de aeropuertos de la base } \\
\text { de datos SKYTRAX }\end{array}$ \\
\hline Geetha et al. & 2017 & Hoteles & $\begin{array}{l}\text { Comentarios de hoteles } \\
\text { de categoría bugdet y } \\
\text { Premium }\end{array}$ \\
\hline Kim et al. & 2017 & Destino & $\begin{array}{l}19,835 \text { comentarios de } \\
\text { Paris de www.virtualtou- } \\
\text { rist.com }\end{array}$ \\
\hline Ren y Hong & 2017 & $\begin{array}{l}\text { Atracciones turís- } \\
\text { ticas }\end{array}$ & $\begin{array}{l}2000 \text { comentarios de } \\
\text { www.ctrip.com de las } 10 \\
\text { atracciones turísticas chi- } \\
\text { nas más importantes }\end{array}$ \\
\hline Lee et $a l$. & 2017 & Hoteles & $\begin{array}{l}20.668 \text { de Tripadvisor. } \\
\text { com revisiones online de } \\
488 \text { hoteles en New York }\end{array}$ \\
\hline
\end{tabular}

Como se puede observar en la tabla 2, la investigación previa es heterogénea tanto en las fuentes de datos como en el sector de análisis, por lo que los resultados no se pueden considerar concluyentes ni extrapolables a distintos contextos. 
Así, el uso de estos métodos todavía está poco desarrollado y en la mayoría de los casos requiere de un alto análisis manual o bien un alto nivel de programación (Chen et al., 2017; Chao y Tand, 2018). Sin embargo, es clara la importancia que su análisis tiene para los destinos y las empresas turísticas, más allá de la evaluación que los mismos puedan hacer de los atributos habituales de medición de calidad de servicio. Las razones se han explicado con anterioridad. Los turistas viajan y van dejando su huella a través de las redes sociales en todos los estadios del mismo (Alonso-Almeida y Ribeiro, 2015,2018). Estos datos son compartidos con su red social o en abierto para cualquier persona y como consecuencia se puede formar la imagen del destino o empresa y tomar sus decisiones de viaje y consumo.

Por lo tanto como advierten Chen et al. (2017, pp 1072) “Las revisiones en línea de los destinos nos permiten capturar lo que los turistas piensan sobre ciertos destinos. La información publicada en las redes sociales es espontáneo y no solicitado, y no está dirigido por una encuesta. Por lo tanto, las revisiones en línea destacan por lo que realmente los viajeros están preocupados, y nos permiten reconocer la importancia relativa de cualquier destino en particular y sus atributos".

Otra tecnología que puede sacar un gran partido del big data y podría tener una gran influencia en el turismo es la inteligencia artificial. De acuerdo con Delamater (2017) en los años ochenta surgió lo que se podría considerar un antecedente de la inteligencia artificial y que es muy popular en nuestros días: los sistemas automáticos de atención al cliente. Estos sistemas redirigen al cliente al departamento con el que quieren contactar pulsando la tecla que le indican, pero no son inteligentes en el sentido que no proveen asistencia como hacen Siri, Cortana o Alexa.

Los actuales asistentes virtuales inteligentes pueden ayudar a cada visitante a una web que necesite información mediante proveer unas respuestas predeterminadas y redirigiendole a donde corresponda en los casos que no tenga la respuesta adecuada. Estos agentes virtuales tienen la habilidad de poder comunicarse en lenguajes pseudonatural. De hecho, esta tecnología está siendo desarrollada en algunas empresas del sector como Expedia o Booking (Hosteltur, 2017).

Así, la inteligencia artificial consiste en interpretar el mundo como los humanos lo hacen, aprendiendo a medida que actúan. Mediante el uso de algoritmos matemáticos, patrones de datos y otra información pueden aprender relaciones complejas y tomar decisiones (Jones et al., 2018). En lugar de programar a la máquina para que haga una tarea, la maquina puede "entrenarse" a si misma a desarrollar una determinada tarea.

La adopción sistemas basados en inteligencia artificial en turismo, al igual que la robótica, podría tener profundas consecuencias en el sector del turismo en términos de empleo, operaciones y calidad del servicio entre otros.

\subsection{Realidad Virtual}

En la actualidad los nuevos modelos económicos se apoyan en poner en valor las emociones frente a la pura racionalidad. Esta nueva realidad económica se conoce como la Economía de la Experiencia. De acuerdo con Petkus (2004), las empresas ya no venden ni productos ni servicios sólo experiencias y para que éstas sean de verdad memorables y produzcan un efecto profundo y duradero en el consumidor se deben 
cumplir dos premisas: 1) Involucrar al cliente en la construcción de la experiencia y 2) que todas las personas, procesos y operaciones de la organización contribuyan al desarrollo de la experiencia.

En los viajes, la posibilidad de poder experimentar previamente se limita a las imágenes del destino que se pueden observar a través de catálogos o videos y, aunque las redes sociales nos permiten recoger las sensaciones de otros viajeros y su valoración, esa experiencia sigue sin ser propia sino basada en opiniones y experiencias ajenas que incluso pueden ser contradictorias en algunos casos. Por eso, la Realidad Virtual (RV) representa una oportunidad real de capitalizar la naturaleza única de los viajes y destinos con el fin de hacer partícipe al cliente de la experiencia del viaje desde el primer contacto inicial y hacer que pueda construir su propia experiencia.

En la actualidad mientras que la RV está siendo aplicada ampliamente en el sector de defensa, al sector médico especialmente en el campo de la educación y al sector de los juegos, en otros sectores como la arqueología o en el ámbito cultural de los museos su desarrollo está en una fase temprana y sólo se han producido algunos casos experimentales con alguna participación española, pero muy escasa especialmente en museos (Bruno et al., 2010).

La RV puede ser hiperrealista y permite interactuar con el mundo virtual que se presenta, de ahí que algunos autores visionarios como Carrozino y Bergamasco (2010), dijesen que es una de las tecnologías más atractivas y potencialmente más efectiva para el desarrollo de la economía de la experiencia, pero la realidad es que es raro encontrar esa tecnología aplicada al turismo en todo el mundo, no sólo en España, más allá de algunos contenidos como fotografías de $360^{\circ}$ o proyectos empresariales experimentales a medida.

Aunque no hay una definición única y consensuada de lo que es la RV, Carrozino y Bergamasco (2010) dicen que la RV es una tecnología compleja que explota otras tecnologías -gráficos 3D, fotografía, robótica y otras- para crear un entorno virtual en el cual los usuarios se encuentren totalmente inmersos dentro donde pueden interactuar. De esta definición se desprenden dos características principales: 1. Inmersión: sensación de estar dentro de un espacio no virtual, sensación que se logra por medio de interfaces sensoriales y 2. Interacción: capacidad del usuario para actuar en ese entorno y recibir retroalimentación de su comportamiento. Ambos elementos juntos hacen pensar que el usuario está realmente en un espacio determinado viviendo una experiencia. Así, siguiendo a Gibersón et al. (2017) definimos la RV como una tecnología innovadora que transporta a la persona a un nuevo ambiente en el que la persona, no está físicamente presente, pero siente que está dentro de ese ambiente a través de la estimulación de múltiples sentidos y que le permite interactuar y recibir una respuesta.

Hay distintos tipos acercamiento a la realidad virtual dependiendo del tipo de dispositivo que se utilice. Cada tipo de dispositivo mismo provee distinto nivel de inmersión, interacción y presencia y a medida que la tecnología de RV se continúa desarrollando el tamaño, dimensiones, material y funcionalidad mejora la experiencia sustancialmente. Giberson et al. (2017) incluye entre las experiencias de RV, las que se desarrollan a través de entornos 3D en el ordenador; entornos digitales con avatares como Second Life o Facebook Spaces donde la persona puede inventar experiencia, interactuar con sus amigos o visitar sitios; usando herramientas inmersivas como gafas, guantes u otros artefactos 
tecnológicos. A medida que la tecnología de VR avanza se provee experiencias más inmersivas con visión, sonidos y sensación de tacto (Desai et al., 2014).

Así, cuanto más inmersiva es la RV los usuarios sienten que su experiencia ha sido más placentera y significativa. Los sistemas inmersivos, además, de proporcionar una experiencia global mejorada envuelve más a los usuarios lo que les permite incrementar su habilidad para absorber conceptos e información en lo que se denomina una experiencia multisensiorial mediada por medios computacionales.

Como se ha mencionado, aunque algunos autores pioneros hablaban en los años 90 de un desarrollo muy alto de la RV en transporte y turismo, las experiencias en este ámbito se pueden considerar marginales. De hecho, la revisión de la literatura arroja muy poca investigación en este ámbito que se podría resumir en tres campos: 1. teórica (Williams y Hobson, 1995; Cheong, 1995; Guttentag, 2010; Martins et al., 2017; Giberson et al., 2017); 2. Empírica basada en realidad aumentada en museos (Dieck y Junk, 2017) o destinos (Tussyadiah y Pesonen, 2016); 3. Empírica basada en tecnología 3D o videos de 360 grados (Griffin et al., 2017) y 4. Empírica basada en juegos (Watcharasukarn et al., 2012; Jeng et al., 2017).

Sin embargo, la RV podría tener un gran impacto en el turismo a través de ciertos ámbitos. En primer lugar, la RV inmersiva constituye un medio para reconstruir los espacios que el tiempo y otras circunstancias han destruido o dañado, de forma que se puedan recuperar para su visualización y divulgación. En este sentido es una herramienta muy útil para el sector de viajes y el turismo en sentido amplio.

Como profetizaron Cheong y Williams y Hobson en 1995 en sendos artículos teóricos que se podrían considerarse pioneros en tratar este tema desde la perspectiva de los viajes y el turismo. En los mismos, estos autores pronostican que la RV podría llegar a ser 1. Una herramienta de marketing para incrementar la visualización del destino; 2. Un medio para reducir los impactos negativos del turismo y 3 . Un camino para explorar "formas distintas de viaje. Por lo tanto, la RV podría ser utilizada tanto en el nivel macro económico como meso y micro. En el nivel macro podría ayudar a la planificación del desarrollo turístico del destino, la construcción de su imagen y su visibilidad. En el nivel meso podría contribuir al desarrollo ordenado y sostenible de los distintos sectores dentro del destino. En el nivel micro puede permitir a los clientes la oportunidad de experimentar un destino o producto turístico y sus atractivos antes de desplazarse al mismo.

Así, el viajero sería capaz de sentir el destino o producto turístico antes de hacer el viaje lo que permitiría: 1. Explorar el destino en mayor profundidad; 2. Hacer que la calidad percibida sea superior a la esperada y 3. Incrementar el deseo que disfrutar del producto o destino. De hecho, la RV es una técnica de visualización que superpone información digitalizada con el objeto de incorporar a la persona en un entorno que mejora su capacidad cognitiva y hace que lo aprecie como si fuera real.

Por otra parte, hay distintos productos turísticos como el turismo cultural, enológico o de aventura entre otros que requieren mucha información para poder apreciar realmente lo que significa la experiencia. Además, en la actualidad, las estructuras existentes no están intactas y, como consecuencia, el periodo histórico vivido no es factiblemente tangible. El patrimonio cultural sufre además de degradación natural que puede ser agravado por la afluencia de turistas. Por tanto, la RV inmersiva permite explorar y apreciar estos sitios 
más allá del tiempo y del espacio e incluso más allá de otras barreras como el idioma. Como consecuencia la RV inmersiva puede mejorar el conocimiento histórico y geográfico y el acercamiento sensorial permite disfrutar de una experiencia turística distinta en un destino específico.

\section{CONCLUSIONES}

En este artículo se ha hecho una aproximación al uso de los robots; el big data y la inteligencia artificial y la realidad virtual en el sector del turismo en sentido amplio. De este análisis se puede extraer algunas conclusiones, que se detallan a continuación.

Primero, aunque en las últimas décadas las tecnologías de la información e internet ha cambiado sustancialmente el sector del turismo, alcanzando altos niveles de automatización en algunas actividades, el uso de robots está en un estadio preliminar. Sin embargo, al igual que ha ocurrido en la industria manufacturera, los pronósticos aseguran que el uso de robots se va a convertir en algo usual en las próximas décadas. Por lo tanto, parece urgente tanto para el sector como para los organismos públicos identificar las oportunidades y amenazas que su adopción presenta, así como las fortalezas y debilidades que cada empresa tiene en este ámbito.

Segundo, el big data puede ser una herramienta para mejorar la competitividad de las empresas turísticas ya que permite analizar tanto las necesidades reales como latentes de los turistas y poder adaptar las operaciones a las mismas. Igualmente, ayuda a conocer mejor a cada cliente de la empresa turística, permitiendo un nivel de personalización de las operaciones para cada cliente que puede contribuir a su satisfacción, lealtad y recompra.

Tercero, la inteligencia artificial está siendo utilizada a través de agentes virtuales para labores de atención al cliente, aunque puede tener otras muchas aplicaciones todavía por explotar. La inteligencia artificial junto con la robótica son un campo de investigación que se desarrollará sustancialmente en los próximos años.

Finalmente, la realidad virtual es otra tecnología con múltiples aplicaciones en el sector de los viajes y el turismo, que se encuentra en un estado incipiente como ya se ha explicado. A medida que los precios de las herramientas de RV inmersiva (cámaras, gafas y otros elementos que permiten la inmersión) reduzcan su precio, su uso y aplicación se puede adoptar más rápidamente (Dieck et al., 2017). Por eso, lo que hace años parecía imposible hoy gracias a la tecnología de RV inmersiva puede ser una realidad con un crecimiento esperado muy alto en ámbitos como el marketing experiencial; la accesibilidad universal; la venta de productos turísticos; el ocio y la formación (AlonsoAlmeida, 2018).

Resumiendo, se presenta un futuro para el sector que va a diferir de lo que se ha conocido hasta ahora y donde la realidad, de nuevo, puede superar a la ficción. Como Santos (2017, pp. 26) avisa "En un futuro cercano las personas vamos a convivir con robots, bots, androides y otras formas de inteligencia artificial cada vez más sofisticadas", lo que va a suponer "una nueva revolución". Así, se han abiertos múltiples líneas de investigación y retos para las empresas en los próximos años y puesto que estas tecnologías pueden tener un alcance global, será necesario involucrar a todos los stakeholders implicados en la toma de decisiones de este ámbito. 


\section{REFERENCIAS}

ACEMOGLU, D. y RESTREPO, P. (2018): “Artificial Intelligence, Automation and Work" (No. w24196), National Bureau of Economic Research. Working Paper 24196. http://www.nber.org/papers/w24196. Revisado el 18 de marzo de 2018.

ALONSO-ALMEIDA, M.M. y RIBEIRO, C. (2018): "Use of Social Media within tourism travel behaviour", Final Conference Social Networks and Travel Behaviour. Milan, February 14th-16th 2018.

ALONSO-ALMEIDA, M.M.; RIBEIRO, C. (2016): "Use of social networks on travel decision making", Social Networks and Travel Behaviour 5MC \& WGs meetings, Bucharest, 18th-19th 2016.

ALONSO ALMEIDA, M.M. (2018): El nuevo materialismo del siglo XXI: Luces y sombras, Colección Real Academia Europea de Doctores. Barcelona.

BOWEN, J. y WHALEN, E. (2017): “Trends that are changing travel and tourism”, Worldwide Hospitality and Tourism Themes, 9 (6), 592-602.

CAPRIELLO, A., MASON, P.R., DAVIS, B. y CROTTS, J.C. (2013): "Farm tourism experiences in travel reviews: A cross-comparison of three alternative methods for data analysis", Journal of Business Research, 66 (6), 778-785.

CARROZZINO, M. y BERGAMASCO, M. (2010): "Beyond virtual museums: Experiencing immersive virtual reality in real museums", Journal of Cultural Heritage, 11 (4), 452-458.

CHAO, A.F. y YANG, H.L. (2018): "Using Chinese radical parts for sentiment analysis and domain-dependent seed set extraction", Computer Speech \& Language, 47, 194213.

CHEN, F.W., GUEVARA PLAZA, A. y ALARCÓN URBISTONDO, P. (2017): “Automatically extracting tourism-related opinion from Chinese social media", Current Issues in Tourism, 20 (10), 1.070-1.087.

CHEONG, R. (1995): “The virtual threat to travel and tourism”, Tourism Management, 16 (6), 417-422.

DELAMATER, N.A. (2017): Brief History of Artificial Intelligence and How It's Revolutionizing Customer Service Today. Available in https://images.g2crowd.com/uploads/ attachment/file/73099/expirable-direct-uploads_2F469f2619-a917-446d-b2b8-14cf8f8 d2c4e_2FChatBotWhitePaper2017.pdf Disponible el 17 de marzo de 2018.

DESAI, P.R., DESAI, P.N., AJMERA, K.D. y MEHTA, K. (2014); A review paper on oculus rift-a virtual reality headset, International Journal of Engineering Trends and Technology, 13 (4), 175-179.

DIECK, M.C. y JUNG, T.H. (2017): "Value of augmented reality at cultural heritage sites: A stakeholder approach", Journal of Destination Marketing \& Management, 6 (2), 110-117.

DUAN, W., YU, Y., CAO, Q. y LEVY, S. (2016): "Exploring the impact of social media on hotel service performance: A sentimental analysis approach", Cornell Hospitality Quarterly, 57 (3), 282-296.

DUNIS, C.L., MIDDLETON, P.W., KARATHANASOPOLOUS, A. y THEOFILATOS, K.A. (Eds.). (2017): Artificial Intelligence in Financial Markets: Cutting Edge Appli- 
cations for Risk Management, Portfolio Optimization and Economics. London: Palgrave Macmillan.

GAN, Q., FERNS, B.H., YU, Y. y JIN, L. (2017): “A text mining and multidimensional sentiment analysis of online restaurant reviews", Journal of Quality Assurance in Hospitality \& Tourism, 18 (4), 465-492.

GEETHA, M., SINGHA, P. y SINHA, S. (2017): "Relationship between customer sentiment and online customer ratings for hotels-An empirical analysis", Tourism Management, 61, 43-54.

GIBERSON, J.; GRIFFIN, T.; DODDS, R. (2017): "Virtual Reality and Tourism: Will the Future of Travel be Virtual?", HTMResearch Working Paper No. 2017/1.

GITTO, S.; MANCUSO, P. (2017): "Improving airport services using sentiment analysis of the websites", Tourism Management Perspectives, 22, 132-136.

GRIFFIN, T., GIBERSON, J., LEE, S.H., GUTTENTAG, D., KANDAUROVA, M., SERGUEEVA, K. y DIMANCHE, F. (2017): "Virtual reality and implications for destination marketing", In 48th Annual Travel and Tourism Research Association (TTRA), International Conference, 20-23 June 2017.

GONZÁLEZ-RODRÍGUEZ, M.R., MARTÍNEZ-TORRES, R. y TORAL, S. (2016): "Post-visit and pre-visit tourist destination image through eWOM sentiment analysis and perceived helpfulness", International Journal of Contemporary Hospitality Management, 28 (11), 2609-2627.

GUTTENTAG, D.A. (2010): "Virtual reality: Applications and implications for tourism", Tourism Management, 31 (5), 637-651.

FUNDACIÓN COTEC (2017): Percepción social de la innovación en España. Informe del estudio cualitativo. http://informecotec.es/media/PRESENTACION-ESTUDIODEMOSCOPICO_IMAGEN-COTEC_VFINAL.pdf. Disponible el 16 de marzo de 2018.

HERTZFIELD, E. (2016): Will robots ever replace guestroom mini bars. Disponible en: www.hotelmanagement.net/tech/will-robots-ever-replace-guestroom-minibars. Revisado el 18 de marzo de 2018.

HOSTELTUR (2017): Inteligencia artificial al servicio de la personalización del servicio. Publicado el 13 de enero de 2017. Disponible en: https://www.hosteltur.com/119905_ inteligencia-artificial-al-servicio-personalizacion-servicio.html. Revisado el 18 de marzo de 2018.

IVANOV, S., WEBSTER, C. y BEREZINA, K. (2017): “Adoption of Robots and Service Automation by Tourism and Hospitality Companies", Revista Turismo \& Desenvolvimento, 27/28, 1.501-1.517. Disponible en SSRN: https://ssrn.com/abstract=2964308. Revisado el 18 de marzo de 2018.

IVANOV, S. y WEBSTER, C. (2017): “Adoption of robots, artificial intelligence and service automation by travel, tourism and hospitality companies - a cost-benefit analysis". International Scientific Conference "Contemporary tourism - traditions and innovations”, 19-21 October 2017, Sofia University.

JENG, M.Y., PAI, F.Y. y YEH, T.M. (2017): “The virtual reality leisure activities experience on elderly people", Applied Research in Quality of Life, 12 (1), 49-65. 
JONES, L.D., GOLAN, D., HANNA, S. y RAMACHANDRAN, M. (2018): “Artificial intelligence, machine learning and the evolution of healthcare: A bright future or cause for concern?", Bone \& Joint Research, 7 (3), 223-225.

KIM, K., PARK, O.J., YUN, S. y YUN, H. (2017): "What makes tourists feel negatively about tourism destinations? Application of hybrid text mining methodology to smart destination management", Technological Forecasting and Social Change, 123, 362369.

KUO, C., CHEN, L. y TSENG, C. (2017): "Investigating an innovative service with hospitality robots", International Journal of Contemporary Hospitality Management, 29 (5), 1305-1321.

LEE, M., JEONG, M. y LEE, J. (2017): “Roles of negative emotions in customers' perceived helpfulness of hotel reviews on a user-generated review website: a text mining approach", International Journal of Contemporary Hospitality Management, 29 (2), 762-783.

MANERO, C.B., GONZÁLEZ, M.G., UCEDA, M.E.G. y GRIJALBA, J.M.M. (2011): "La influencia de las TIC en la estructura del sistema de distribución turístico", Cuadernos de Turismo, 28, 9-22.

MARTINS, J., GONÇALVES, R., BRANCO, F., BARBOSA, L., MELO, M. y BESSA, M. (2017): “A multisensory virtual experience model for thematic tourism: A Port wine tourism application proposal", Journal of Destination Marketing \& Management, 6 (2), 103-109.

PHILANDER, K. y ZHONG, Y. (2016): “Twitter sentiment analysis: capturing sentiment from integrated resort tweets", International Journal of Hospitality Management, 55, 16-24.

TUSSYADIAH, I.P. y PESONEN, J. (2016): "Impacts of peer-to-peer accommodation use on travel patterns", Journal of Travel Research, 55(8), 1022-1040.

MAURER, M., GERDES, J.C., LENZ, B. y WINNER, H. (Eds.) (2016): Autonomous driving: technical, legal and social aspects. Berlin, Heidelberg: Springer Open.

MELENDEZ-FERNANDEZ, F., GALINDO, C. y GONZALEZ-JIMENEZ, J. (2017): "A web-based solution for robotic telepresence", International Journal of Advanced Robotic Systems, 14(6), 1729881417743738.

PETKUS, E. (2004): "Enhancing the application of experiential marketing in the arts", International Journal of Nonprofit and Voluntary Sector Marketing, 9(1), 49-56.

REN, G. y HONG, T. (2017): "Investigating Online Destination Images Using a TopicBased Sentiment Analysis Approach", Sustainability, 9 (10), 1765.

RESEARCH AND MARKETS (2017): “Collaborative Robots Market: Global Industry Analysis, Trends, Market Size and Forecasts up to 2023", Report.

RODRÍGUEZ ANTÓN, J.M., ALONSO ALMEIDA, M.M., RUBIO ANDRADA, L. y ESTEBAN ALBERDI, C. (2008): "Conocimiento y aprendizaje en las grandes cadenas hoteleras españolas en Iberoamérica: Internet como herramienta de aprendizaje organizativo", Cuadernos de Turismo, 21, 135-157.

SANTOS, M.J. (2017): "Regulación legal de la robótica y la inteligencia artificial: retos de futuro= Legal regulation of robotics and artificial intelligence: future challenges", Revista Jurídica de la Universidad de León, 4, 25-50. 
TIMMS, M.J. (2016): "Letting artificial intelligence in education out of the box: educational cobots and smart classrooms", International Journal of Artificial Intelligence in Education, 26 (2), 701-712.

WATCHARASUKARN, M., PAGE, S. y KRUMDIECK, S. (2012): "Virtual reality simulation game approach to investigate transport adaptive capacity for peak oil planning", Transportation Research Part A: Policy and Practice, 46 (2), 348-367.

WILLIAMS, P. y HOBSON, J.P. (1995): "Virtual reality and tourism: fact or fantasy?", TOURISM MANAGEMENT, 16 (6), 423-427.

ZHANG, Z., YE, Q., ZHANG, Z. y LI, Y. (2011): "Sentiment classification of Internet restaurant reviews written in Cantonese", Expert Systems with Applications, 38 (6), 7.674-7.682. 